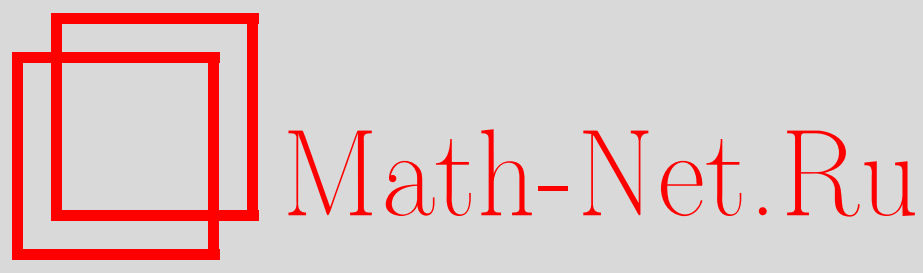

У. Бруццо, В. Н. Рубцов, Когомологии косоголоморфных алгеброидов Ли, ТМФ, 2010, том 165, номер 3, 426439

DOI: https://doi.org/10.4213/tmf6586

Использование Общероссийского математического портала Math-Net.Ru подразумевает, что вы прочитали и согласны с пользовательским соглашением http://www . mathnet.ru/rus/agreement

Параметры загрузки:

IP: 35.173 .137 .237

26 апреля 2023 г., $12: 36: 23$

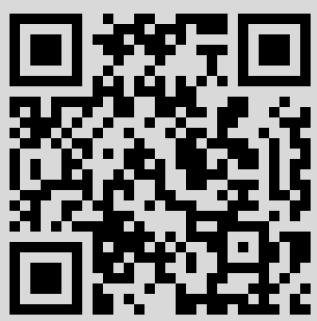




\title{
ФИЗИКА
}

Том 165, № 3

декабрь, 2010

2010 г.

\author{
У. Бруццо*, В. Н. Рубцов ${ }^{\ddagger}$
}

\section{КОГОМОЛОГИИ КОСОГОЛОМОРФНЫХ АЛГЕБРОИДОВ ЛИ}

\begin{abstract}
Введено понятие косоголоморфного алгеброида Ли на комплексном многообразии и исследованы некоторые теории когомологий, которые можно связать с этим объектом. Приводятся примеры и приложения вводимых понятий в терминах разного рода голоморфных структур Пуассона.
\end{abstract}

Ключевые слова: голоморфные алгеброиды Ли, скрученные произведения алгеброидов Ли, когомологии алгеброидов Ли, голоморфные когомологии Пуассона.

\section{1. ВВЕДЕНИЕ}

Комплексная структура комплексного многообразия $X$ служит богатым источником разнообразных теорий когомологий, например когомологий Дольбо или голоморфных когомологий де Рама, которые нетривиальным образом связаны с обычными когомологиями де Рама $X$. Комплексную структуру на $X$ можно рассматривать как интегрируемое разложение

$$
T_{X} \otimes \mathbb{C}=T_{X}^{1,0} \oplus T_{X}^{0,1}
$$

с условием $\overline{T_{X}^{1,0}}=T_{X}^{0,1}$.

С другой стороны, рассматривается понятие алгеброида Ли; говоря упрощенно(точное определение приводится ниже), имеется отображение векторных расслоений $a: A \rightarrow T_{X}$ вместе с “поднятием" структуры алгебры Ли на сечениях $T_{X}$ до структуры алгебры Ли на сечениях $A$. Тогда, естественно, можно думать также и о поднятии разложения (1). В настоящей статье мы изучаем теории когомологий, связанные с подобными структурами. В частности, мы рассматриваем алгеброиды Ли, получаемые с помощью скрученного произведения (технические определения и смысл этого понятия будут уточнены в основном тексте статьи) голоморфного

\footnotetext{
*International School for Advanced Studies, Trieste, Italy
}

${ }^{\dagger}$ National Institute of Nuclear Physics, Sezione di Trieste, Italy. E-mail: bruzzo@sissa.it

${ }^{\ddagger}$ Université d’Angers, Département de Mathématiques LAREMA, VFR Sciences, Angers, France

${ }^{\S}$ Институт теоретической и экспериментальной физики, Москва, Россия. E-mail: Volodya.Roubtsov@univ-angers.fr 
алгеброида Ли $\mathscr{A}_{1}$ с комплексно-сопряженным другого голоморфного алгеброида Ли $\mathscr{A}_{2}$. Мы называем полученную таким образом структуру косоголоморфным алгеброидом Ли. Частным случаем этой конструкции является пример, разобранный в статье [1], где $\mathscr{A}_{2}$ является голоморфным касательным расслоением $X$.

Настоящая работа посвящена изучению теории когомологий косоголоморфных алгеброидов. В разделе 3 после напоминания базовых определений в разделе 2 мы рассматриваем известные понятия представления алгеброида Ли, скрученного произведения алгеброидов Ли и вводим новые понятия почти комплексной структуры на алгеброиде Ли и косоголоморфного алгеброида Ли. В разделе 4 мы формулируем и доказываем основную теорему о свойствах когомологий таких алгеброидов Ли. В разделе 5 приведены некоторые примеры, относящиеся, главным образом, к различным когомологиям, связанным с голоморфными пуассоновыми тензорами.

\section{2. ПРЕДВАРИТЕЛЬНЫЕ СВЕДЕНИЯ}

Алгеброиды Ли. Начнем с напоминания понятий алгеброида Ли и когомологий алгеброидов Ли.

Пусть $M$ - гладкое многообразие, $T_{M}$ - его касательное расслоение, и пусть $\mathfrak{X}(M)$ обозначает пространство векторных полей на многообразии $M$, снабженное обычной скобкой Ли - коммутатором $[\cdot, \cdot]$.

ОПредЕЛЕНиЕ 1. Алгеброид $A$ над многообразием $M$ - это векторное расслоение на $M$ вместе с морфизмом векторных расслоений $a: A \rightarrow T_{M}$ (называемым якорем) и структурой алгебры Ли на пространстве глобальных сечений $\Gamma(A)$ такое, что:

1) $a: \Gamma(A) \rightarrow \mathfrak{X}(M)$ есть гомоморфизм алгебр Ли;

$2)$ для всех сечений $\alpha, \beta \in \Gamma(A)$ и любых гладких функций $f$ выполняется следующее правило Лейбница:

$$
\{\alpha, f \beta\}=f\{\alpha, \beta\}+a(\alpha)(f) \beta
$$

(мы обозначаем $\{\cdot, \cdot\}$ скобку в $\Gamma(A))$.

Алгеброид Ли $A$ называется транзитивным, если его якорь $а$ сюръективен.

Морфизмы между двумя алгеброидами Ли $(A, a)$ и $\left(A^{\prime}, a^{\prime}\right)$ над одним и тем же многообразием $M$ определяются естественным образом, т.е. это морфизмы векторных расслоений $\phi: A \rightarrow A^{\prime}$ такие, что отображение $\phi: \Gamma(A) \rightarrow \Gamma\left(A^{\prime}\right)$ есть гомоморфизм алгебр Ли, и диаграмма

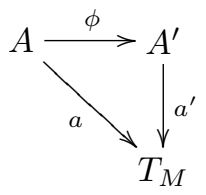

коммутативна.

ПримеР 1. Интересным примером транзитивного алгеброида Ли является алгеброид Amъи векторного расслоения $E$ на $M$. Это расслоение $\mathscr{D}(E)$ дифференциальных операторов первого порядка на $E$, символ которых - скалярная матрица. Якорь $\sigma: \mathscr{D}(E) \rightarrow T_{M}$ есть отображение символа. Более того, $\operatorname{ker}(\sigma) \simeq \operatorname{End}(E)$. 
С каждым алгеброидом Ли $A$ можно связать коцепной комплекс $\left(C_{A}^{\bullet}, \delta\right), C_{A}^{\bullet}=$ $\Gamma\left(\Lambda^{\bullet} A^{*}\right)$, и дифференциал $\delta$ определяется как [2]

$$
\begin{aligned}
(\delta \xi)\left(\alpha_{1}, \ldots, \alpha_{p+1}\right)= & \sum_{i=1}^{p+1}(-1)^{i-1} a\left(\alpha_{i}\right)\left(\xi\left(\alpha_{1}, \ldots, \widehat{\alpha}_{i}, \ldots, \alpha_{p+1}\right)\right)+ \\
& +\sum_{i<j}(-1)^{i+j} \xi\left(\left\{\alpha_{i}, \alpha_{j}\right\}, \ldots, \widehat{\alpha}_{i}, \ldots, \widehat{\alpha}_{j}, \ldots, \alpha_{p+1}\right),
\end{aligned}
$$

если $\xi \in C_{A}^{p}$ и $\alpha_{i} \in \Gamma(A), 1 \leqslant i \leqslant p+1$. Соответствующие когомологии обозначаются $H^{\bullet}(A)$ и называются когомологиями алгеброида Ли $A$.

Подобное определение можно дать и в случае комплексного алгеброида Ли, когда $A$ является комплексным векторным расслоением, а $T_{M}$ заменяется своей комплексификацией $T_{M} \otimes \mathbb{C}$. Аналогично имеем понятие голоморфного алгеброида Ли на комплексном многообразии $X$, где $A$ - голоморфное векторное расслоение (которое мы будем обозначать $\mathscr{A}), T_{M}$ заменяется голоморфным касательным расслоением $\Theta_{X}$, и мы требуем, чтобы $\mathscr{A}$ допускал структуру пучка алгебр Ли, удовлетворяющих соответствующему правилу Лейбница.

\section{3. КОСОГОЛОМОРФНЫЕ АЛГЕБРОИДЫ ЛИ}

Напомним некоторые результаты, касающиеся когомологий голоморфных алгеброидов Ли. Рассматриваемая ниже теория обобщает конструкцию, предложенную в работе [1]. Нам представляется полезным и интересным рассмотреть это расширение, хотя мы и не будем его использовать в полной общности.

Пусть $X-n$-мерное компактное комплексное многообразие, $\Theta_{X}$ - его голоморфное касательное расслоение, $T_{X}$ - его касательное расслоение, когда $X$ рассматривается как $2 n$-мерное вещественное гладкое многообразие. $\Omega_{X}^{i}$ обозначает расслоение голоморфных $i$-форм на $X$.

3.1. Почти комплексные алгеброиды Ли. Существует очень естественный способ расширить понятие почти комплексного многообразия до понятия почти комплексного алгеброида Ли (это обобщение определения почти комплексного пуассонова многообразия, введеного в статье [3], где приведены также некоторые примеры). Пусть $M$ - почти комплексное многообразие с почти комплексной структурой $J_{M}: T_{M} \rightarrow T_{M}$.

ОПРЕДЕЛЕНИЕ 2. Почти комплексная структура $J_{A}$ на вещественном алгеброиде Ли $A \stackrel{a}{\rightarrow} T_{M}$ - это эндоморфизм векторного расслоения $J_{A}: A \rightarrow A$ такой, что $J_{A}^{2}=-\operatorname{id}_{A}$ и $J_{M} \circ a=a \circ J_{A}$.

Как обычно, имеется разложение

$$
A \otimes \mathbb{C}=A^{1,0} \oplus A^{0,1}
$$

соответственно собственным значениям $\pm i$ структуры $J_{A}$. Мы положим

$$
\lambda_{A}^{p, q}=\Lambda^{p}\left(A^{*}\right)^{1,0} \oplus \Lambda^{q}\left(A^{*}\right)^{0,1} .
$$


Обозначим также $C_{A}^{p, q}=\Gamma\left(\lambda_{A}^{p, q}\right)$; дифференциал $d_{A}$ комплекса $C_{A}^{\bullet}=\bigoplus_{p, q} C_{A}^{p, q}$ разлагается в сумму

$$
d_{A}=\partial_{A}^{\prime}+\partial_{A}+\bar{\partial}_{A}+\partial_{A}^{\prime \prime}
$$

где

$$
\begin{array}{ll}
\partial_{A}^{\prime}: C_{A}^{p, q} \rightarrow C_{A}^{p+2, q-1}, & \partial_{A}: C_{A}^{p, q} \rightarrow C_{A}^{p+1, q}, \\
\bar{\partial}_{A}: C^{p, q} \rightarrow C^{p, q+1}, & \partial_{A}^{\prime \prime}: C^{p, q} \rightarrow C^{p-1, q+2}
\end{array}
$$

и выполняются тождества

$$
\begin{gathered}
\partial_{A}^{2}=\left[\partial_{A}^{\prime}, \bar{\partial}_{A}\right], \quad \bar{\partial}_{A}^{2}=\left[\partial_{A}^{\prime \prime}, \partial_{A}\right], \quad\left[\partial_{A}, \partial_{A}^{\prime \prime}\right]+\left[\partial_{A}, \bar{\partial}_{A}\right]=0 \\
\left(\partial_{A}^{\prime}\right)^{2}=\left(\partial_{A}^{\prime \prime}\right)^{2}=\left[\partial_{A}, \partial_{A}^{\prime}\right]=\left[\bar{\partial}, \partial_{A}^{\prime \prime}\right]=0 .
\end{gathered}
$$

Дифференциальный комплекс $\left(C_{A}^{\bullet}, d_{A}\right)$ допускает (регулярную) фильтрацию

$$
F_{p} C_{A}^{k}=\bigoplus_{\substack{r+s=k \\ r \geqslant p-k}} C_{A}^{r, s} .
$$

Непосредственное обобщение анализа, выполненного в работе [4], показывает, что справедливо следующее предложение.

ПреДЛОжЕниЕ 1. Спектральная последователъность, ассочиированная с фильтрачией (3) дифференицального комплекса $\left(C_{A}^{\bullet}, d_{A}\right)$, сходится к комплексифицированным когомологиям алгеброида Ли $A$, m.е. к когомологиям $H^{\bullet}(A) \otimes \mathbb{C}$.

Пусть $X-n$-мерное комплексное многообразие.

ОПРЕДЕЛЕНИЕ 3. Почти комплексная структура на алгеброиде Ли $A$ над $X$ называтся интегрируемой, если существует голоморфный алгеброид Ли $\mathscr{A}$ такой, что:

1) $A^{1,0} \simeq \mathcal{C}_{X}^{\infty} \otimes \mathcal{O}_{X} \mathscr{A}$ как пучки $\mathcal{C}_{X}^{\infty}$-модулей;

2) при этом изоморфизме скобка на $A \otimes \mathbb{C}$ ограничивается до скобки на $\mathscr{A}$;

3) якорь $a: A \otimes \mathbb{C} \rightarrow T_{X}^{\mathbb{C}}$ совпадает с $\tilde{a}$ на $\mathscr{A}$.

В этом случае мы будем называть $\mathscr{A}$ голоморфной структурой на $A$. Заметим, что дифференциал $d_{A}$ алгеброида Ли $A$ и $\partial_{A}$ на $\mathscr{A}$ связаны соотношениями

$$
\begin{aligned}
d_{A}^{1,0}(f \otimes \xi) & =f \otimes \partial_{A} \xi+a^{*}(\partial f) \wedge \xi \\
d_{A}^{0,1}(f \otimes \xi) & =a^{*}(\bar{\partial} f) \wedge \xi
\end{aligned}
$$

если $f$ - гладкая функция и $\xi \in \Lambda^{\bullet} \mathscr{A}^{*}$.

Интегрируемость почти комплексной структуры $J_{A}$ на алгеброиде Ли может быть, как обычно, проверена с помощью подходящего тензора Нийенхейса. Можно определить элемент $N_{A} \in \Gamma\left(\Lambda^{2}\left(A^{*}\right) \otimes A\right)$,

$$
N_{A}(\alpha, \beta)=\{\alpha, \beta\}+J_{A}\left\{J_{A} \alpha, \beta\right\}+\left\{\alpha, J_{A} \beta\right\}-\left\{J_{A} \alpha, \beta\right\},
$$


и показать, что структура $J_{A}$ интегрируема тогда и только тогда, когда $N_{A}=0$.

Предполагая, что $A$ допускает комплексную структуру $\mathscr{A}$, положим $\Omega_{\mathscr{A}}^{k}=\Lambda^{k} \mathscr{A}^{*}$ и обозначим через $\partial_{\mathscr{A}}$ дифференциал алгеброида Ли $\mathscr{A}$. Мы получаем комплекс пучков на $X$

$$
\Omega_{\mathscr{A}}^{0} \stackrel{\partial_{\mathscr{A}}}{\rightarrow} \Omega_{\mathscr{A}}^{1} \stackrel{\partial_{\mathscr{A}}}{\rightarrow} \Omega_{\mathscr{A}}^{2} \cdots .
$$

Пусть $\partial_{\mathscr{A}}, d_{A}$ - дифференциалы алгеброидов Ли $\mathscr{A}$ и $A$, положим $\Omega_{\mathscr{A}}^{\bullet}=\Lambda^{\bullet} \mathscr{A}^{*}$. Получаем инъективное отображение $\Omega_{\mathscr{A}}^{\bullet} \hookrightarrow \Lambda^{\bullet} A^{*} \otimes \mathbb{C}$.

ЛЕмма 1. Если А допускает голоморфную структуру $\mathscr{A}, \operatorname{mo~}_{A}$ ограничивается до $\partial_{A} н а \Omega_{\mathscr{A}}^{\bullet}$.

ДокАЗАТЕЛЬСтво. Если $f$ - голоморфная функция, а $\alpha \in \Gamma(\mathscr{A})$, то

$$
d_{A}(f)(1 \otimes \alpha)=a(1 \otimes \alpha)(f)=\tilde{a}(\alpha)(f)=\partial_{A}(f)(\alpha)
$$

так что утверждение справедливо в степени 0. Если $\xi \in \Gamma\left(\Omega_{\mathscr{A}}^{1}\right)$, то

$$
\begin{aligned}
d_{A}(1 \otimes \xi)(1 \otimes \alpha, 1 \otimes \beta) & =\tilde{a}(\alpha)(\xi(\beta))-\tilde{a}(\beta)(\xi(\alpha))-\xi(\{\alpha, \beta\})= \\
& =\left(1 \otimes \partial_{A} \xi\right)(1 \otimes \alpha, 1 \otimes \beta),
\end{aligned}
$$

так что в степени 1 оно также выполнено. Применение правила Лейбница завершает доказательство.

\section{2. Касательный комплекс регулярного голоморфного алгеброида Ли.} Скажем, что голоморфный алгеброид Ли $a: \mathscr{A} \rightarrow \Theta_{X}$ регулярен, если его якорь $a$ имеет постоянный ранг всюду на $X$. В этом случае образ $\mathscr{D}$ на $\mathscr{A}$ в $\Theta_{X}$ является инволютивным голоморфным подрасслоением в $\Theta_{X}$, поточечно касательным к регулярному голоморфному слоению $\mathscr{F}$ в $X$. Дифференциал $\partial_{A}$ комплекса пучков $\Lambda^{\bullet} \mathscr{A}^{*}$ ограничивается до дифференциала $\partial_{\mathscr{D}}: \Lambda^{\bullet} \mathscr{D}^{*} \rightarrow \Lambda^{\bullet+1} \mathscr{D}^{*}$.

ПредлОЖение 2. 1. Ядро $\partial_{\mathscr{D}}: \mathcal{O}_{X} \rightarrow \mathscr{D}^{*}$ естъ пучок $\mathcal{O}_{\mathscr{F}}$ голоморфных функиий на $X$, локально-постоянных вдоль листов $\mathscr{F}$.

2. Комплекс пучков $\left(\Lambda^{\bullet} \mathscr{D}^{*}, \partial_{\mathscr{D}}\right)$ является точным в положительных степенях.

3. Существует изоморфизм $H^{k}\left(X, \mathcal{O}_{\mathscr{F}}\right) \simeq \mathbb{H}^{k}\left(X, \Lambda^{\bullet} \mathscr{D}^{*}\right)$ для всех $k \geqslant 0$.

Здесь $\mathbb{H}^{\bullet}$ обозначает функтор гиперкогомологий.

ДокАЗАТЕЛЬство. Пусть $\Omega_{\mathscr{F}}^{\bullet}$ обозначает фактор голоморфного комплекса де Рама $\left(\Omega_{X}^{\bullet}, \partial\right)$ по ядру сопряженного $a^{*}$ к отображению якоря. Для всех $k$ назовем $\Omega_{\mathscr{F}}^{k}$ пучком $\mathscr{F}$-слоеных голоморфных дифференииальных форм на $X$.

Существует слоеный $\partial$-оператор $\partial_{\mathscr{F}}: \Omega_{\mathscr{F}}^{\bullet} \rightarrow \Omega_{\mathscr{F}}^{\bullet+1}$, и оказывается, что сопряженный к якорю задает изоморфизм комплексов $\left(\Lambda^{\bullet} \mathscr{D}^{*}, \partial_{\mathscr{D}}\right) \simeq\left(\Omega_{\mathscr{F}}^{\bullet}, \partial_{\mathscr{F}}\right)$. Поэтому мы можем доказать точность комплекса $\left(\Omega_{\mathscr{F}}^{\bullet}, \partial_{\mathscr{F}}\right)$ (в положительных степенях).

Мы можем также ввести пучки $\Omega_{\mathscr{F}}^{p, q}$ гладких $\mathscr{F}$-слоеных дифференциальных форм $X$ ходжева типа $(p, q)$ (согласно стандартному определению). Имеем дифференциал $\bar{\partial}_{\mathscr{F}}: \Omega_{\mathscr{F}}^{\bullet, \bullet} \rightarrow \Omega_{\mathscr{F}}^{\bullet, \bullet+1}$. 
Далее, существуют голоморфные координаты $\left(z^{1}, \ldots, z^{m}, y^{1}, \ldots, y^{n-m}\right)$ вокруг каждой точки $X$, где $n=\operatorname{dim}_{\mathbb{C}} X$ и $m=\operatorname{rk}_{\mathbb{C}} \mathscr{D}$, так что листы $\mathscr{F}$ задаются уравнениями $y^{i}=$ const, и $z$ - координаты на листах. Поскольку точность комплекса пучков $\left(\Omega_{\mathscr{F}}^{\bullet}, \partial_{\mathscr{F}}\right)$ - вопрос локальный, мы можем предположить, что $X=Z \times Y$, и отождествить листы $\mathscr{F}$ с комплексными подмногообразиями $Z \times\{y\}$ для $y \in Y$. Далее $\left(z^{1}, \ldots, z^{m}\right)$ и $\left(y^{1}, \ldots, y^{n-m}\right)$ являются локальными коодинатами в $Z$ и $Y$ соответственно. Заметим, что в этих координатах сечения $\Omega_{\mathscr{F}}^{k}$ записываются как

$$
\eta=\sum \eta_{i_{1}, \ldots, i_{k}}(z, y) d z^{i_{1}} \wedge \cdots \wedge d z^{i_{k}}
$$

тогда как $\Omega_{\mathscr{F}}^{p, q}$ записываются как

$$
\tau=\sum \tau_{i_{1}, \ldots, i_{p}, j_{1}, \ldots, j_{q}}(z, \bar{z}, y) d z^{i_{1}} \wedge \cdots \wedge d z^{i_{p}} \wedge d \bar{z}^{j_{1}} \wedge \cdots \wedge d \bar{z}^{j_{q}}
$$

Утверждение 1 теперь получается непосредственно. Более того, теперь легко показать, что естественное отображение $\Omega_{\mathscr{F}}^{\bullet} \rightarrow \Omega_{\mathscr{F}}^{\bullet \bullet}$ является резольвентой. Стандартный результат гомологической алгебры (см., например, лемму 8.5 в монографии [5]) показывает, что комплекс $\Omega_{\mathscr{F}}^{\bullet}$ квазиизоморфен комплексу гладких комплекснозначных $\mathscr{F}$-слоеных дифференциальных форм, и, поскольку последний является точным (см. с. 215 в монографии [6]), то точным (в положительных степенях) является и первый. Это доказывает утверждение 2. Утверждение 3 является непосредственным следствием двух предыдущих.

3.3. Представления алгеброидов Ли. Представление алгеброида Ли $\mathrm{A} A \stackrel{a}{\rightarrow}$ $T_{M}$ в векторном расслоении $E$ есть морфизм алгеброидов Ли $\nabla: A \rightarrow \mathscr{D}(E)$, где $\mathscr{D}(E) \stackrel{\sigma}{\rightarrow} M$ - алгеброид Атьи $E$ [7], [8]. Поэтому, если $\alpha, s-$ сечения $A$ и $E$ соответственно, $\nabla(\alpha)$ действует на $s$; мы обозначим это действие через $\nabla_{\alpha} s$. Предыдущее абстрактное определение означает что $\nabla$ удовлетворяет условиям

$$
\nabla_{\{\alpha, \beta\}}=\left[\nabla_{\alpha}, \nabla_{\beta}\right], \quad \sigma(\nabla(\alpha))=a(\alpha)
$$

Когда имеется представление $A$ в $E$, скажем, как обычно, что $E$ является $A$-модулем.

Можно определить когомологии алгеброида Ли с коэффициентами в $E$, рассматривая "твистованный" комплекс $C_{A}^{\bullet}(E)=\Gamma\left(\Lambda^{\bullet} A^{*} \otimes E^{*}\right)$ и определяя дифференциал согласно

$$
\begin{aligned}
\left(\delta_{E} \xi\right)\left(\alpha_{1}, \ldots, \alpha_{p+1}, s\right)= & \sum_{i=1}^{p+1}(-1)^{i-1}\left[a\left(\alpha_{i}\right) \xi\left(\alpha_{1}, \ldots, \widehat{\alpha}_{i}, \ldots, \alpha_{p+1}, s\right)-\right. \\
& \left.-\xi\left(\alpha_{1}, \ldots, \widehat{\alpha}_{i}, \ldots, \alpha_{p+1}, \nabla_{\alpha_{i}} s\right)\right]+ \\
& +\sum_{i<j}(-1)^{i+j} \xi\left(\left\{\alpha_{i}, \alpha_{j}\right\}, \ldots, \widehat{\alpha}_{i}, \ldots, \widehat{\alpha}_{j}, \ldots, \alpha_{p+1}, s\right) .
\end{aligned}
$$


3.4. Скрученные произведения алгеброидов Ли. Нам потребуется понятие скрученного произведения алгеброидов Ли [1], [9]-[12]. Мы приведем его для случая вещественных алгеброидов, но подобные конструкции могут быть приведены и для гладкого комплексного или голоморфного случаев.

Скажем, что $A$ и $B$ образуют скрученное произведение, если $A$ является $B$-модулем, $B$ является $A$-модулем и справедливы соотношения

$$
\begin{gathered}
{[a(\alpha), b(\beta)]=-a\left(\nabla_{\beta} \alpha\right)+b\left(\nabla_{\alpha} \beta\right),} \\
\nabla_{\alpha}\left\{\beta_{1}, \beta_{2}\right\}=\left\{\nabla_{\alpha} \beta_{1}, \beta_{2}\right\}+\left\{\beta_{1}, \nabla_{\alpha} \beta_{2}\right\}+\nabla_{\nabla_{\beta_{2}} \alpha} \beta_{1}-\nabla_{\nabla_{\beta_{1}} \alpha} \beta_{2}, \\
\nabla_{\beta}\left\{\alpha_{1}, \alpha_{2}\right\}=\left\{\nabla_{\beta} \alpha_{1}, \alpha_{2}\right\}+\left\{\alpha_{1}, \nabla_{\beta} \alpha_{2}\right\}+\nabla_{\nabla_{\alpha_{2}} \beta} \alpha_{1}-\nabla_{\nabla_{\alpha_{1}} \beta} \alpha_{2} .
\end{gathered}
$$

Если $A, B$ образуют скрученное произведение алгеброидов Ли, то их прямая сумма $A \oplus B$ может быть наделена структурой алгеброида Ли $A \bowtie B$ с помощью якоря $c$, определенного как $c(\alpha+\beta)=a(\alpha)+b(\beta)$, и скобки

$$
\left\{\alpha_{1}+\beta_{1}, \alpha_{2}+\beta_{2}\right\}=\left(\left\{\alpha_{1}, \alpha_{2}\right\}+\nabla_{\beta_{1}} \alpha_{2}-\nabla_{\beta_{2}} \alpha_{1}\right)+\left(\left\{\beta_{1}, \beta_{2}\right\}+\nabla_{\alpha_{1}} \beta_{2}-\nabla_{\alpha_{2}} \beta_{1}\right) .
$$

Если эти условия выполнены, мы можем рассматривать когомологии $A$ с коэффициентами в $A$-модуле $\Lambda^{\bullet} B^{*}$ и, точно так же, когомологии $B$ с коэффициентами в $B$-модуле $\Lambda^{\bullet} A^{*}$.

Довольно громоздкие условия (6) могут быть изящно переписаны в терминах коммутирования двух дифференциалов этих комплексов [1]. Таким образом, в случае скрученного произведения алгеброидов Ли мы получаем двойной комплекс. Более того, в работе [1] показано, что когомология тотального комплекса изоморфна когомологии скрученного произведения $A \bowtie B$ (с тривиальными коэффициентами).

3.5. Косоголоморфные алгеброиды Ли. В работе [1] доказано, что любой голоморфный алгеброид Ли на комплексном многообразии $X$ может образовывать скрученное произведение с алгеброидом Ли $T_{X}^{0,1}$, и с использованием этого факта развита теория когомологий голоморфных алгеброидов Ли. Минимальными дополнительными усилиями мы можем обобщить эту теорию и изучить комплексы алгеброидов Ли, полученных скрученными произведениями голоморфных алгеброидов Ли с антиголоморфными. Мы называем это косоголоморфной структурой. Разумеется, это обобщает случай комплексификации касательного расслоения $T_{X, \mathbb{C}}$. Мы развиваем здесь элементы теории когомологий для этого класса алгеброидов Ли.

ОПРЕДЕЛЕНИЕ 4. Комплексный алгеброид Ли $A \stackrel{a}{\rightarrow} T_{X}^{\mathbb{C}}$ на комплексном многообразии $X$ наделен косоголоморфной структурой, если

1) существует скрученное произведение алгеброидов Ли $A_{1}, A_{2}$ такое, что $A \simeq$ $A_{1} \bowtie A_{2}$;

2) $A_{1} \simeq \mathcal{C}^{\infty} \otimes \mathscr{A}_{1}$ и $\bar{A}_{2} \simeq \mathcal{C}^{\infty} \otimes \mathscr{A}_{2}$ (как комплексные алгеброиды Ли) для некоторых голоморфных алгеброидов Ли $\mathscr{A}_{1}, \mathscr{A}_{2}$.

Отметим, что из этих условий следует, что для якорей $a_{1}, a_{2}$ алгеброидов $A_{1}, A_{2}$ можно записать $a_{1}\left(A_{1}\right) \subset T^{1,0} X, a_{2}\left(A_{2}\right) \subset T^{0,1} X$. 
ЗАмечание 1. Если $\mathscr{A}$ - голоморфный алгеброид Ли, то $A_{1}=\mathcal{C}_{X}^{\infty} \otimes_{\mathcal{O}_{X}} \mathscr{A}$ и $T_{X}^{0,1}$ образуют скрученное произведение. Поэтому мы получаем комплексный алгеброид Ли $A=A_{1} \bowtie T_{X}^{0,1}$ с косоголоморфной структурой. Это воспроизводит теорию, развитую в работе [1], которая, таким образом, является специальным случаем нашей теории. Если в дополнение к этому $\mathscr{A}$ есть голоморфное касательное расслоение $\Theta_{X}$, то $A$ - комплексификация $T_{X}^{\mathbb{C}}$, и наша теория когомологий совпадает с теорией де Рама. В более общем случае, если дан комплексный алгеброид Ли $A=A_{1} \bowtie A_{2}$ с косоголоморфной структурой, якорь $a_{2}: A_{2} \rightarrow T^{0,1} X$ определяет морфизм комплексных алгеброидов Ли $A \rightarrow A_{1} \bowtie T^{0,1} X$. В свою очередь это определяет морфизмы $H^{\bullet}\left(A_{1} \bowtie T^{0,1} X\right) \rightarrow H^{\bullet}(A)$.

\section{4. ЛОКАЛЬНЫЕ КОГОМОЛОГИИ КОСОГОЛОМОРФНЫХ АЛГЕБРОИДОВ ЛИ}

Пусть $A$ - косоголоморфный алгеброид Ли на комплексном многообразии $X$. Рассмотрим пучки

$$
\lambda_{A}^{p, q}=\Lambda^{p} A_{1}^{*} \otimes \Lambda^{q} A_{2}^{*}
$$

с дифференциалами

$$
\partial_{A}: \lambda_{A}^{p, q} \rightarrow \lambda_{A}^{p+1, q}, \quad \bar{\partial}_{A}: \lambda_{A}^{p, q} \rightarrow \lambda_{A}^{p, q+1}
$$

Поскольку комплексные алгеброиды Ли $A, A_{2}$ образуют скрученное произведение, $\left(\lambda^{\bullet} \bullet, \partial_{A}, \bar{\partial}_{A}\right)$ является двойным комплексом [1]. Следующий результат можно легко проверить (см. также работу [1], предложение 4.6).

ПреДЛОЖЕНИЕ 3. Когомологии алгеброида Ли А изоморфны когомологиям тотального комплекса двойного комплекса $\left(\Gamma\left(\lambda^{\bullet \bullet \bullet}\right), \partial_{A}, \bar{\partial}_{A}\right)$.

Обозначим через $\mathscr{A}$ пучок голоморфных сечений алгеброида $A_{1}$. Более того, мы скажем, что $A_{2}$ транзитивен, если $a_{2}: A_{2} \rightarrow T_{X}^{0,1}$ сюръективно.

ЛЕмма 2. Если алгеброид А снабжен косоголоморфной структурой и $A_{2}$ транзитивен, то $\operatorname{ker}\left[\lambda_{A}^{p, 0} \stackrel{\bar{\partial}_{A}}{\rightarrow} \lambda_{A}^{p, 1}\right] \simeq \Omega_{\mathscr{A}}^{p}$.

ДокАЗАТЕльство. Если $f-$ некоторая функция, то $\bar{\partial}_{A}(f)(\alpha)=a_{2}(\alpha)(f)$ для $\alpha \in \Gamma\left(\lambda_{A}^{p, 1}\right)$; если $\bar{\partial}_{A}(f)=0$ и $a_{2}$ сюръективно, то функция $f$ голоморфна. Пусть $\beta=\sum_{i} \alpha_{i} \otimes f_{i}$ - сечение $\lambda_{A}^{p, 0}$. Мы можем предположить, что $\alpha_{i}$ голоморфны (именно, они являются сечениями $\left.\Omega_{\mathscr{A}}^{p}\right)$. Если $\bar{\partial}_{A} \beta=0$, то $\sum_{i} \alpha_{i} \otimes \bar{\partial}_{A} f_{i}=0$, откуда следует, что функции $f_{i}$ являются голморфными. Тогда $\beta=\sum_{i} f_{i} \alpha_{i} \otimes 1$ есть сечение $\Omega_{A}^{p}$. 
В общем случае, без предположения транзитивности $A_{2}$, пусть $\mathscr{K}^{p}=\operatorname{ker}\left[\lambda_{A}^{p, 0} \stackrel{\bar{\partial}_{A}}{\rightarrow}\right.$ $\left.\lambda_{A}^{p, 1}\right]$. Поскольку $\bar{\partial}_{A}\left(\Omega_{\mathscr{A}}^{0}\right)=\bar{\partial}_{A}\left(\mathcal{O}_{X}\right)=0$ и $A_{1} \simeq \mathcal{C}^{\infty} \otimes \mathscr{A}$, имеем инъективное отображение комплексов $\Omega_{\mathscr{A}}^{\bullet} \rightarrow \mathscr{K}^{\bullet}$. Получаем следующую диаграмму:

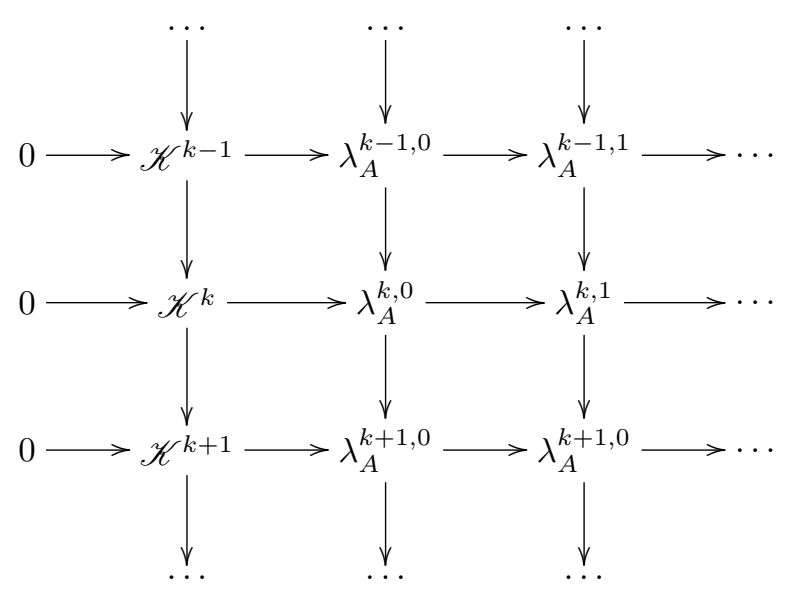

ОПредЕЛЕНИЕ 5. Будем говорить, что алгеброид $A$ удовлетворяет $\partial_{A}$-лемме Пуанкаре $\left(\bar{\partial}_{A}\right.$-лемме Пуанкаре), если для всех $p$ комплекс пучков $\left(\lambda_{A}^{\bullet, p}, \partial_{A}\right)$ (соответственно $\left.\left(\lambda_{A}^{p, \bullet}, \bar{\partial}_{A}\right)\right)$ точен в положительных степенях.

Пример 2. Комплексный алгеброид Ли из замечания 1 удовлетворяет $\bar{\partial}_{A}$-лемме Пуанкаре, которая в данном случае означает просто точность комплекса Дольбо, "твистованного" голоморфным расслоением $\mathscr{A}$. Более того, в этом случае $A_{2}$ очевидно транзитивен.

Следующая теорема описывает главные когомологические свойства комплексного алгеброида Ли, снабженного косоголоморфной структурой.

Теорема. Пусть алгеброид А удовлетворяет $\bar{\partial}_{A}$-лемме Пуанкаре. Тогда справедливы следующие утверждения.

1. Обобщенная голоморфная теорема де Рама. Существует изоморфизм

$$
\mathbb{H}^{p}\left(X, \mathscr{K}^{\bullet}\right) \simeq H^{p}(A)
$$

где $\mathbb{H}^{\bullet}$ обозначает гиперкогомологии.

2. Обобщенная теорема Дольбо. Существует изоморфизм

$$
H^{p}\left(X, \mathscr{K}^{q}\right) \simeq H^{p}\left(\Gamma\left(\lambda_{A}^{q, \bullet}\right), \bar{\partial}_{A}\right),
$$

где $H^{p}\left(X, \mathscr{K}^{q}\right)$ обозначают когомологию пучков.

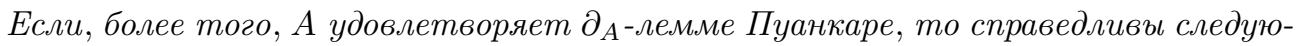
щие утверждения.

3. Комплекс пучков $\left(\Lambda^{\bullet} A^{*}, d_{A}\right)$ точен в положительных степенях (т.е. существует лемма Пуанкаре для дифференциала $\left.d_{A}\right)$. 
4. Обобщенная теорема де Рама. Существует изоморфизм $H^{p}(X, \mathscr{F} \infty) \simeq H^{p}(A)$, где $H^{p}\left(X, \mathscr{F}^{\infty}\right)$ - когомологии пучков, и $\mathscr{F}^{\infty}=\operatorname{ker}\left[\mathcal{C}_{X}^{\infty} \stackrel{d_{A}}{\rightarrow} A^{*}\right]$ является пучком функций Казимира $А$.

5. Комплекс пучков $\left(\mathscr{K}^{\bullet}, \partial_{A}\right)$ точен в положительных степенях.

Наконец, если в дополнение $к$ предьдущим условиям $A_{2}$ является транзитивным, то справедливы следующие утверждения.

6. Существует изоморфизм $H^{p}(X, \mathscr{F}) \simeq H^{p}(A)$, где $\mathscr{F}=\operatorname{ker}\left[\mathcal{O}_{X} \stackrel{\partial_{A}}{\rightarrow} \mathscr{A}^{*}\right]-$ пучок функиий Казимира $\mathscr{A}$.

7. Если многообразие $X$ штейново, то группъ когомологий $H^{p}(X, \mathscr{F})(u$, следовательно, группы $\left.H^{p}(A)\right)$ изоморфны группам когомологий комплекса глобальных сечений $\Omega_{\mathscr{A}}^{\bullet}$.

ДокАЗАТЕльство. 1. Член $E_{1}$ первой спектральной последовательности двойного комплекса пучков $\left(\lambda^{\bullet} \bullet \bullet, \partial_{A}, \bar{\partial}_{A}\right)$ имеет вид

$$
\begin{aligned}
& E_{1}^{p, 0} \simeq \mathscr{K}^{p}, \\
& E_{1}^{p, q}=0 \quad \text { для } \quad q>0 .
\end{aligned}
$$

Следовательно, спектральная последовательность вырождается на первом шаге, и мы получим

$$
\begin{aligned}
& E_{2}^{p, 0} \simeq \mathscr{H}^{p}\left(\mathscr{K}^{\bullet}\right), \\
& E_{2}^{p, q}=0 \quad \text { для } \quad q>0,
\end{aligned}
$$

что вместе с предложением 3 доказывает, что композиция $\mathscr{K}^{\bullet} \hookrightarrow \lambda_{A}^{\bullet, 0} \hookrightarrow \Lambda^{\bullet} A^{*}$ является квазиизоморфизмом между комплексами $\left(\Lambda^{\bullet} A^{*}, d_{A}\right)$ и $\mathscr{K}^{\bullet}$. Поскольку пучок $\Lambda^{\bullet} A^{*}$ тонкий, это доказывает утверждение 1 теоремы.

Утверждение 2 следует из абстрактной теоремы де Рама.

Утверждение 3 очевидно.

$\Lambda^{\bullet} A^{*}$ является (тонкой) резольвентой $\mathscr{F}^{\infty}$, так что абстрактная теорема де Рама доказывает утверждение 4. Утверждение 5 следует из утверждения 3 и квазиизоморфизма $\left(\Lambda^{\bullet} A^{*}, d_{A}\right) \simeq \mathscr{K}^{\bullet}$.

Так как алгеброид $A_{2}$ транзитивен, то имеем $\mathscr{K}^{\bullet} \simeq \Omega_{\mathscr{A}}^{\bullet}$. С другой стороны, согласно утверждению 5 комплекс, образованный $\mathscr{F}$ в степени 0 , квазиизоморфен $\Omega_{\mathscr{A}}^{\bullet}$. Поэтому

$$
H^{p}(X, \mathscr{F}) \simeq \mathbb{H}^{p}\left(X, \Omega_{\mathscr{A}}^{\bullet}\right) \simeq H^{p}(A),
$$

т.е. справедливо утверждение 6.

Поскольку комплекс $\Omega_{\mathscr{A}}^{\bullet}$ является резольвентой $\mathscr{F}$, то существует спектральная последовательность со вторым членом $E_{2}^{p, q}=H^{q}\left(H^{p}\left(X, \Omega_{\mathscr{A}}^{\bullet}\right), \partial_{A}\right)$, которая сходится к $H^{\bullet}(X, \mathscr{F})$. Если многообразие $X$ штейново, то единственные ненулевые компоненты во втором члене - это $E_{2}^{0, q}=H^{q}\left(\Omega_{\mathscr{A}}^{\bullet}(X), \partial_{A}\right)$, откуда и следует утверждение 7.

ЗАмечАниЕ 2. 1. Отметим, что в утверждениях 1-5 теоремы предположение о транзитивности $A_{2}$ нам не требуется. 
2. Если $A_{2}=T^{0,1} X$ с $a_{2}=$ id (см. замечание 1 ), то $\mathscr{K} \bullet \simeq \Omega_{\mathscr{A}}^{\bullet}$, и утверждение 1 теоремы дает результат из работы [1] (меняя в [1] утверждение "когомологии комплекса $\Omega_{\mathscr{A}}^{\bullet}$ " (нулевые в положительных степенях) на "гиперкогомологии комплекса $\left.\Omega_{\mathscr{A}}^{\bullet} "\right)$.

3. Если $A_{1}=T^{1,0} X, a_{1}=\mathrm{id}$, так что $A$ - комплексный алгеброид де Рама, утверждение 2 теоремы превращается в теорему Дольбо, утверждение 4 - в теорему де Рама, утверждение 1 - в голоморфную теорему де Рама [5].

\section{5. ПРИМЕРЫ}

5.1. Голоморфные структуры Пуассона. Этот пример был рассмотрен в работе [1], однако для полноты мы кратко опишем его здесь. Пусть $X$ - комплексное многообразие и $P$ - голоморфный пуассонов тензор, так что голоморфное кокасательное расслоение $\Omega_{X}^{1}$ с якорем $P: \Omega_{X}^{1} \rightarrow \Theta_{X}$ является голоморфным алгеброидом Ли. Как отмечалось выше, можно рассмотреть скрученное произведение этого алгеброида с алгеброидом Ли, естественно ассоциированным с расслоением $T_{X}^{0,1}$, получив комплексный алгеброид Ли с косоголоморфной структурой $A$. Доказанная выше теорема и лемма 2 доставляют изоморфизмы

$$
\mathbb{H}^{p}\left(X, \mathscr{V}_{P}^{\bullet}\right) \simeq H^{p}(A), \quad H^{p}\left(X, \mathscr{V}_{P}^{q}\right) \simeq H^{p}\left(\Gamma\left(\lambda_{A}^{q, \bullet}\right), \bar{\partial}_{A}\right)
$$

где $\mathscr{V}_{P}^{\bullet}$ - комплекс пучков голоморфных поливекторных полей с дифференциалом, задаваемым пуассоновым тензором $P$. Первый из этих изоморфизмов описывает соотношение между когомологиями голоморфного пуассонова многообразия и когомологиями подлежащего гладкого пуассонова многообразия.

Если многообразие $X$ штейново, гиперкогомологии $\mathbb{H}^{\bullet}\left(X, \mathscr{V}_{P}^{\bullet}\right)$ изоморфны когомологиям комплекса глобальных сечений $\mathscr{V}_{P}^{\bullet}$.

5.2. Голоморфные касательные когомологии Лихнеровича-Пуассона. Пусть $P$ - регулярный голоморфный тензор Пуассона на комплексном многообразии $X$ (т.е. ранг линейного комплексного отображения $P_{x}:\left(\Theta_{X}^{*}\right)_{x} \rightarrow\left(\Theta_{X}\right)_{x}$ не зависит от $x$ ). Положив $\mathscr{A}=\Omega_{X}^{1} / \operatorname{ker} P$, получаем точную последовательность голоморфных векторных расслоений

$$
0 \rightarrow \operatorname{ker} P \rightarrow \Omega_{X}^{1} \rightarrow \mathscr{A} \rightarrow 0
$$

Более того, скобка, определяемая $P$ на локальных сечениях $\Omega_{X}^{1}$, опускается до скобки на $\mathscr{A}$, и мы получаем морфизм $\tilde{a}: \mathscr{A} \rightarrow \Theta_{X}$. Это определяет голоморфный алгеброид Ли $\mathscr{A} \stackrel{\tilde{a}}{\rightarrow} \Theta_{X}$ (разумеется, это специальный случай ситуации, описанной в п. 3.2). Мы назовем гиперкогомологии ассоциированного комплекса $\Omega_{\mathscr{A}}^{\bullet}$ голоморфными касательными когомологиями Лихнеровича-Пуассона.

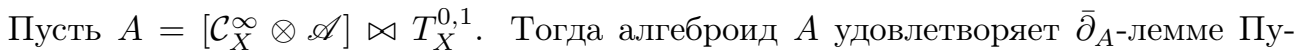
анкаре, а $A_{2}$ транзитивен. Более того, согласно предложению 2 алгеброид $A$ удовлетворяет $\partial_{A}$-лемме Пуанкаре, так что он удовлетворяет всем условиям теоремы. 
Поэтому мы получаем изоморфизмы

$$
\mathbb{H}^{p}\left(X, \Omega_{\mathscr{A}}^{\bullet} \simeq H^{p}(X, \mathscr{F}) \simeq H^{p}(A)\right.
$$

Если $X$ является многообразием Штейна, то эти группы совпадают также с группами $H^{p}\left(\Omega_{\mathscr{A}}^{\bullet}(X), \partial_{A}\right)$.

5.3. Косоголоморфные структуры Пуассона. Предположим, что на комплексном многообразии $X$ заданы два голоморфных пуассоновых тензора $P_{1}$ и $P_{2}$. Векторные расслоения $\Omega_{X}^{1,0}$ и $\Omega_{X}^{0,1}$ снабжены скобками, задаваемыми $P_{1}$ и комплексно-сопряженным пуассоновым тензором $\bar{P}_{2}$ соответственно, и задают структуры комплексных алгеброидов Ли $A_{1}, A_{2}$ с якорями $P_{1}: A_{1} \rightarrow T_{X}^{1,0}, \bar{P}_{2}: A_{2} \rightarrow T_{X}^{0,1}$. Каждый алгеброид имеет представления одного в другом, задаваемые как

$$
\nabla_{\alpha} \beta=\partial_{P_{1}(\alpha)}(\beta), \quad \nabla_{\beta} \alpha=\bar{\partial}_{\bar{P}_{2}(\beta)}(\alpha),
$$

если $\alpha \in \Omega^{1,0}(X), \beta \in \Omega^{0,1}(X)$.

ПреДЛОЖЕНИЕ 4. Алгеброидъ Ли $A_{1}$ u $A_{2}$ вместе с модулъными структурами, заданными уравнением (7), образуют скрученное произведение алгеброидов Ли. Это скрученное произведение $A=A_{1} \bowtie A_{2}$ является алгеброидом Ли с косоголоморфной структурой, а его подлежащее векторное расслоение есть комплексификаиия гладкого кокасательного расслоения $X$.

ДокАЗАТЕЛЬство. Необходимо только проверить выполнение условий скрученного произведения. Как уже отмечалось, согласно предложению 4.5 из работы [1] это превращается в проверку коммутируемости дифференциала $\partial_{1}$ алгеброида Ли $A_{1}$, твистованного с помощью $\Lambda^{\bullet} A_{2}^{*}$, с дифференциалом алгеброида Ли $A_{2}$, твистованного с помощью $\Lambda^{\bullet} A_{1}^{*}$.

Обобщая очевидным образом формулы из предложения 4.25 в работе [1], мы можем записать

$$
\partial_{1}(\mu \otimes \nu)=\left[\left[P_{1}, \mu\right]\right] \otimes \nu+\sum_{i}\left(e_{i} \wedge \mu\right) \otimes \mathcal{L}_{P_{1}\left(e^{* i}\right)} \nu
$$

где $\mu \in \Gamma\left(\Lambda^{\bullet} A_{1}^{*}\right)=\Gamma\left(\Lambda^{\bullet} T_{X}^{1,0}\right), \nu \in \Gamma\left(\Lambda^{\bullet} A_{2}^{*}\right)=\Gamma\left(\Lambda^{\bullet} T_{X}^{0,1}\right),\left\{e_{i}\right\}$ - локальный базис сечений $T_{X}^{1,0},\left\{e^{* i}\right\}$ - двойственный базис, $\mathcal{L}$ - производная Ли, [[ · , · ]] - скобка Схоутена. Аналогично имеем

$$
\partial_{2}(\mu \otimes \nu)=\mu \otimes\left[\left[\bar{P}_{2}, \nu\right]\right]+\sum_{i} \mathcal{L}_{\bar{P}_{2}(f * i)} \mu \otimes\left(f_{i} \wedge \nu\right)
$$

где $\left\{f_{i}\right\}$ - локальный базис сечений $T_{X}^{0,1},\left\{f^{* i}\right\}$ - двойственный базис. Так как оба дифференциала удовлетворяют правилу Лейбница, то достаточно проверить их коммутативность в случае, когда тензор $\mu$ голоморфен, а $\nu$ антиголоморфен. Таким образом, получаем

$$
\partial_{1}(\mu \otimes \nu)=\left[\left[P_{1}, \mu\right]\right] \otimes \nu=d_{1} \mu \otimes \nu,
$$


где $d_{1}$ - дифференциал (нетвистованного) комплекса Лихнеровича-Пуассона $P_{1}$. Аналогично имеем

$$
\partial_{2}(\mu \otimes \nu)=\mu \otimes\left[\left[\bar{P}_{2}, \nu\right]\right]=\mu \otimes d_{2} \nu,
$$

где $d_{2}$ - дифференциал комплекса Лихнеровича-Пуассона $\bar{P}_{2}$. Так как диффференциал $d_{1} \mu$ голоморфен, а $d_{2} \nu$ антиголоморфен, мы получаем

$$
\partial_{1} \partial_{2}(\mu \otimes \nu)=d_{1} \mu \otimes d_{2} \nu=\partial_{2} \partial_{1}(\mu \otimes \nu)
$$

Эти результаты усиливают замечание из работы [3], где отмечено, что скобки Схоутена $P_{1}$ и $\bar{P}_{2}$ обращаются в нуль, т.е. тензоры $P_{1}$ и $\bar{P}_{2}$ удовлетворяют условию бигамильтоновости. Однако, как мы обсуждали в работе [13], условие скрученного произведения сильнее, чем условие бигамильтоновости, и первое влечет второе.

В условиях предложения 4 косоголоморфный алгеброид Ли $A$ не удовлетворяет ни одному из условий теоремы. Если пуассонов тензор $P_{2}$ невырожден, то имеется изоморфизм алгеброидов Ли $\bar{P}_{2}: A_{2} \rightarrow T_{X}^{0,1}$, где $T_{X}^{0,1}$ снабжен стандартной структурой алгеброида Ли. Таким образом, мы получаем пример из п. 5.1.

5.4. Косоголоморфные пуассоновы структуры. Мы можем совместить два предыдущих примера, рассматривая два регулярных голоморфных пуассонова тензора $P_{1}, P_{2}$ и алгеброид Ли скрученного произведения, полученный, как в п. 5.2, из $P_{1}$ и комплексно-сопряженного к $P_{2}$. Таким образом, мы попадаем в ситуацию, когда верны утверждения 1-5 теоремы.

\section{6. ЗАКЛЮЧЕНИЕ}

Развиваемая в настоящей работе теория предлагает одновременно обобщения и упрощения известных результатов и их доказательств, приведенных в работе [1]. Более того, совершенно естественные приложения этой теории обнаруживаются при изучении пар голоморфных пуассоновых структур, удовлетворяющих условиям совместности голоморфных бигамильтоновых систем [13]. Поэтому можно предположить, что наша теория будет полезна при изучении голоморфных интегрируемых систем. Можно применить ее также к изучению комплексных многообразий с заданной на них парой совместных, возможно особых, голоморфных слоений.

Предложенная теория когомологий может также быть использована при изучении теории деформаций комплексных алгеброидов Ли и связи условий деформации с вышеупомянутыми условиями совместности. Пример такой связи был предложен недавно в статье [14], мотивированной некоторыми естественными вопросами классической механики. Наши конструкции и их очевидные обобщения для случая алгеброидов Ли скрученных произведений из работы [14], их двойственных алгеброидов и симплектических реализаций скрученных коалгеброидов предлагают полезный инструментарий и прозрачный язык для изучения "внутренних" деформаций и условий совместности в голоморфном случае. 
Благодарности. Эта статья в основном была написана во время визита У. Бруццо в Физический департамент Университета Ратгерс, который он благодарит за гостеприимство. В.Н. Рубцов выражает благодарность Лаборатории теоретической физики Университета Сержи-Понтуаз за гостеприимство. Часть результатов была представлена В.Н. Рубцовым на конференции "Дифференциальные уравнения и топология" в Институте математики НАН Украины в феврале 2010 г. в Киеве. Автор благодарен проекту CNRS PICS "Проблемы математической физики" (Франция-Украина) за поддержку его визита в Киев. Авторы также благодарны И. Косманн-Шварцбах и М. Стиенону за полезные обсуждения и признательны Университету г. Анжер, Франция, и SISSA за гостеприимство и финансовую поддержку. Работа выполнена при частичной поддержке INFN (проект PI14 "Непертурбативная динамика калибровочных теорий”), а также проекта MISGAM (Методы интегрируемых систем, геометрии, прикладной математики), EINSTEIN Italo-Russian project "Интегрируемость в топологических струнных и полевых теориях", MATPYL Angers-SISSA project "Алгеброиды Ли, эквивариантные когомологии и топологические квантовые и струнные полевые теории".

\section{Список литературы}

[1] C. Laurent-Gengoux, M. Stiénon, P. Xu, Int. Math. Res. Not., 2008 (2008), Art. ID rnn 088, 46 pp., arXiv: 0707.4253.

[2] S. Evens, J.-H. Lu, A. Weinstein, Quart. J. Math. Oxford Ser. (2), 50:200 (1999), 417-436.

[3] L. A. Cordero, M. Fernández, R. Ibáñez, L. Ugarte, Ann. Global Anal. Geom., 18:3-4 (2000), 265-290.

[4] V.V. Lychagin, V.N. Rubtsov, "Non-holonomic filtration: algebraic and geometric aspects of non-integrability", Geometry in Partial Differential Equations, eds. A. Prastaro, Th. M. Rassias, World Sci., River Edge, NJ, 1994, 189-214.

[5] C. Voisin, Hodge Theory and Complex Algebraic Geometry. V. I, Cambridge Stud. Adv. Math., 76, Cambridge Univ. Press, Cambridge, 2007.

[6] I. Vaisman, Cohomology and Differential Forms, Pure Appl. Math., 21, Marcel Dekker, New York, 1973.

[7] В. Н. Рубцов, УМH, 35:4(214) (1980), 209-210.

[8] K. C. H. Mackenzie, General Theory of Lie Groupoids and Lie Algebroids, London Math. Soc. Lecture Note Ser., 213, Cambridge Univ. Press, Cambridge, 2005.

[9] J.-H. Lu, Duke Math. J., 86:2 (1997), 261-304.

[10] K. C. H. Mackenzie, Electron. Res. Announc. Amer. Math. Soc., 4 (1998), 74-87.

[11] T. Mokri, Glasgow Math. J., 39:2 (1997), 167-181.

[12] J. Huebschmann, "Differential Batalin-Vilkovisky algebras arising from twilled Lie-Rinehart algebras", Poisson Geometry, Banach Center Publ., 51, eds. J. Grabowski, P. Urbański, Polish Acad. Sci., Warsaw, 2000, 87-102.

[13] U. Bruzzo, V. Rubtsov, On compatibility of Lie algebroid structures, in preparation.

[14] J. F. Cariñena, J. M. Nuñes da Costa, P. Santos, J. Phys. A, 39:22 (2006), 6897-6918. 